\title{
EDITORIAL
}

\section{Biomarkers and Surrogates}

The development and application of biomarkers in neurodegenerative disorders have become increasingly important to clinical practice and therapeutic trials. Neurodegenerative diseases now form a major focus for drug discovery and development. In this issue, we have emphasized four of the most common and important neurologic diseases that result from neurodegeneration: Alzheimer disease, Parkinson disease, Huntington disease, and amyotrophic lateral sclerosis. Although the clinical manifestations of each vary according to the topography of the neurons that are primarily affected, they share many common features. These include abnormal accumulations of proteins with nerve cell death, a broad range of genetic risk, and a clinical course of progressive deterioration. Symptomatic treatments of variable efficacy are available for each, but there is a distressing lack of treatments that protect neurons from dying or that even retard their degeneration. These four diseases are therefore high-priority targets for developing additional symptomatic and novel neuroprotective therapies: in the aggregate they are prevalent, carry grievous morbidity, and are associated with great emotional and financial costs to both individuals and society. They are also realistic targets: substantial progress has already been made at the basic science level in terms of unraveling the causes and pathophysiology of each disease, developing disease models, and designing novel therapeutic strategies. However, in many of these diseases there are significant limitations in the ability to predict disease onset, to give definitive diagnoses, to measure progression or to detect accurately the effects of therapeutic intervention on purely clinical grounds. Accordingly, in each of these areas there is great interest in developing objective biologically based markers that can be used to predict risk, diagnose, stage, or track the course and treatment of neurodegeneration and thereby complement currently employed clinical measures.

A biomarker should reflect an important and possibly unique feature of the disease. The ideal surrogate endpoint or outcome measure is a laboratory substitute for a clinically meaningful result, and should lie directly in the causal pathway linking disease to outcome. There are three different areas where biomarkers may have important roles to play: as markers of trait, state, and rate. A measure of disease trait is a marker such as a genetic mutation that predicts the likelihood of developing a disease. A measure of disease trait may also indicate susceptibility to disease, for instance cholesterol levels and heart disease risk. A measure of disease state is, in essence, a diagnostic biomarker. As an example, levels of tau are elevated in the CSF of patients with Alzheimer disease and have been proposed as a diagnostic biomarker. In all instances, a diagnostic biomarker should have high sensitivity and specificity (ideally both $90 \%$ or greater) to have a major impact on clinical practice. A measure of disease rate or change is a marker that can be used to track progression of the pathophysiology of the disease, or to detect the effects of a therapeutic intervention. Promising markers in this regard, such as measures of atrophy on quantitative magnetic resonance brain scans, are often referred to as surrogate outcomes or surrogate biomarkers. It is theoretically possible that a single biomarker may fulfill all roles of measuring trait, state, and rate in a particular disease. However, the requirements for a robust predictive marker, a sensitive and specific diagnostic marker to separate diseases, and a validated marker of progression in trials in neurodegenerative brain disease may be quite different and make this possibility unlikely. In practical terms, it is more likely that it will be necessary to use a number of different markers, either separately or in combination, to fulfill these three different roles.

This special issue is aimed at documenting advances in biological markers and consists of a series of commissioned reviews that summarize the current state of genetic, molecular and biochemical measures, and neuroimaging as biomarkers and surrogate outcomes in neurodegenerative diseases. The final paper deals with neuroimaging in multiple sclerosis, which has led the way in developing biomarkers as both diagnostic and surrogate outcome measures. We are extremely grateful to the authors of the 12 papers that comprise this issue of NeuroRx $x^{\circledR}$ for sharing their expertise with the neurotherapeutic community, and we anticipate that their contributions will spur further development in the field of developing, validating, and applying biologic markers and surrogate outcome measures in neurodegenerative diseases.

Nick Fox, M.D., M.R.C.P., Institute of Neurology, Queen Square, London WC1N 3BG, UK John H. Growdon, M.D., Department of Neurology, Massachusetts General Hospital, Boston, MA 02114 